\title{
DISEÑO E IMPLEMENTACIÓN DEL SISTEMA DE ESPECTROSCOPIA DE FOTOIMPEDANCIA ELÉCTRICA EN EL DESARROLLO DE SENSORES ÓPTICOS BASADOS EN MICROVARAS DE ZnO
}

\author{
DESIGN AND IMPLEMENTATION OF AN ELECTRIC PHOTOIMPEDANCE \\ SPECTROSCOPY SYSTEM IN THE DEVELOPMENT OF OPTICAL \\ SENSORS BASED ON ZnO MICRORODS
}

D. A. Garzón-Ramos*, D. A. Guzmán-Embús, D. C. Barrera-Andrade, A. M. Florez-Villamil y C. Vargas-Hernández.

Laboratorio de Propiedades Ópticas de los Materiales

Universidad Nacional de Colombia - Sede Manizales

\section{RESUMEN}

Recibido: 17 de febrero de 2014

Aceptado: 23 de marzo de 2014

E-mail: dagarzonr@unal.edu.co

El creciente interés por la adquisición de nuevo conocimiento acerca del comportamiento de materiales empleados en la construcción de dispositivos optoelectrónicos ha llevado a la búsqueda de nuevas técnicas de instrumentación y caracterización de materiales que permitan comprender fenómenos electrónicos asociados a propiedades ópticas inherentes a materiales semiconductores. Por esta razón se desarrolló una cámara hermética con emisión controlada en el UV-visible e infrarrojo cercano que se integra al impedancimetro Solartron SI 1260 IMPEDANCE/GAIN-PHASE ANALYZER con el objetivo de mejorar y diversificar su funcionamiento; convirtiendo así este equipo en un sistema capaz de realizar medidas de impedancia eléctrica en presencia de diferentes tipos de radiación óptica a través de la técnica de espectroscopia de fotoimpedancia eléctrica. Se realizaron estudios de películas microestructuradas de $\mathrm{ZnO}$ a través del sistema de foto-impedancia eléctrica en temperatura ambiente y a $120^{\circ} \mathrm{C}$. Los resultados indican una disminución de la impedancia en un orden de magnitud para las películas en presencia de radiación $\mathrm{UV}$, hecho asociado a la cercanía de esta radiación con el gap de $3.17 \mathrm{eV}$ calculado para las microvaras de $\mathrm{ZnO}$. Los mejores resultados en los tiempos de saturación y relajación de las películas se lograron a $120^{\circ} \mathrm{C}$. De este modo se logró determinar la alta eficacia de las películas microestructuradas de $\mathrm{ZnO}$ como posibles sensores resistivos de UV cuando se someten a altas temperaturas.

Palabras claves: Foto-impedancia eléctrica, microvaras, sensor UV, ZnO.

\begin{abstract}
The growing interest in the acquisition of new knowledge about the behavior of materials used in the construction of optoelectronic devices has led to the search for new materials instrumentation and characterization techniques for understanding electronic phenomena associated with optical properties inherent to semiconductor materials. For this reason, an airtight chamber with controlled emission in the UV - visible and near infrared was developed. This chamber was integrated to the impedance analyzer Solartron SI 1260 IMPEDANCE / GAIN - PHASE ANALYZER in order to improve and diversify their operation, thus making this equipment in a system capable of carrying out electric impedance measurements in the presence of different types of optical radiation using electrical photoimpedance spectroscopy technique. Microstructured studies of $\mathrm{ZnO}$ films were made at ambient temperature and $120^{\circ} \mathrm{C}$ through the system developed. The results indicate a decrease in impedance in one order of magnitude for the films in the presence of UV radiation. This fact was associated with the proximity of this radiation with the gap of $3.17 \mathrm{eV}$ calculated for $\mathrm{ZnO}$ microrods. The best results in the saturation and relaxation times of the films were obtained at $120{ }^{\circ} \mathrm{C}$. Thus, it was possible to determine the high efficiency of the $\mathrm{ZnO}$ microstructured films as possible $\mathrm{UV}$ resistive sensors under high temperature.
\end{abstract}

Keywords: Electric photoimpedance, microrods, UV sensor, $\mathrm{ZnO}$. 


\section{INTRODUCCIÓN}

El $\mathrm{ZnO}$ es un semiconductor del grupo II-VI con propiedades eléctricas, mecánicas, optoelectrónicas, piezoeléctricas y químicas lo cual le confiere un amplio rango de aplicaciones en transistores [1], diodos rectificadores [2], generadores eléctricos [3], celdas solares [4], sensores de gas [5] y sensores de ADN [6]. Debido a su brecha de energía prohibida de 3.3 $\mathrm{eV}$, lo hace un material ideal para estudiar la sensibilidad ante la radiación UV, promoviendo cambios en sus propiedades. Los detectores UV son usados para monitorear el grosor de la capa de ozono y la radiación solar UV dando un área de investigación para nuevos sensores de mayor sensibilidad, de bajo costo de fabricación, menores dimensiones, lo cual el $\mathrm{ZnO}$ se presenta como un material promisorio para esta labor, al ser fácil de obtener en el laboratorio y presentar una gran variedad de estructuras que pueden ser utilizadas para la fabricación de nanodispositivos.[7] En este trabajo fue realizado un estudio del comportamiento de las propiedades eléctricas de una película estructurada de $\mathrm{ZnO}$ irradiada con luz UV, a través de la espectroscopia de impedancia haciendo modificaciones a la celda para llevar acabo las mediciones de Espectroscopia de Fotoimpedancia Eléctrica.

\section{MATERIALES Y MÉTODOS}

\section{Celda de fotoimpedancia eléctrica.}

La variación de la respuesta de un material con respecto a las excitaciones eléctricas puede verse alterada cuando este sufre los efectos de otros tipos de energía interactuando con él, ya sea incrementando o disminuyendo la intensidad de la misma. En la técnica propuesta de espectroscopia de fotoimpedancia eléctrica se evalúan los cambios del comportamiento eléctrico de los materiales en presencia de diferentes tipos de radiación óptica como UV, visible e infrarrojo cercano; estudios que con temperaturas controladas permiten obtener información relevante para el desarrollo de sensores ópticos.

En la realización de las medidas se emplea una de las configuraciones de medición más utilizadas en espectroscopia de impedancia como son los electrodos en paralelo, en donde una señal eléctrica variable en frecuencia se genera entre los dos electrodos y se mide la respuesta con respecto a la señal de entrada. En la Figura 1 se puede observar la configuración para este tipo de medidas en donde se envía una señal de corriente que circula por la muestra y se toma información sobre la diferencia de potencial entre sus terminales para construir los diagramas de impedancia.

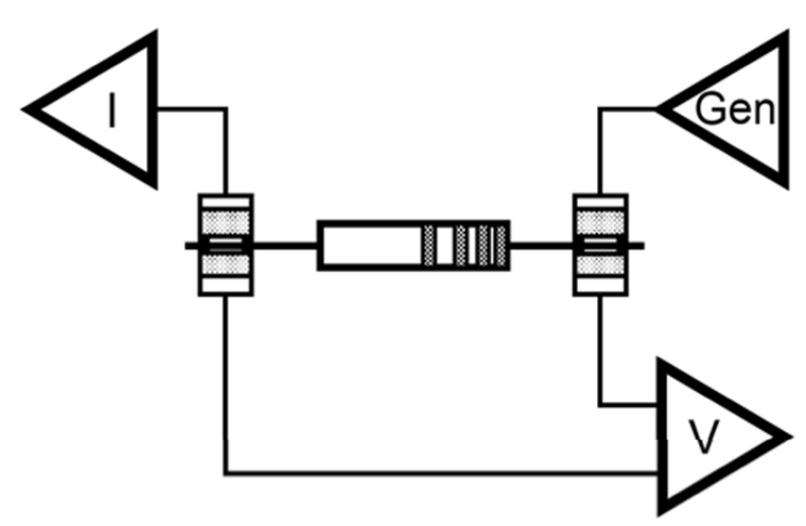

Figura 1. Configuración de electrodos paralelos en la medida de impedancia eléctrica. Medida de corriente (I), medida de tensión (V) y generador de señal (Gen).

Diseño e implementación.

En principio se identificaron las funciones, necesidades y restricciones de la cámara oscura con emisión de radiación óptica controlada, denominada cámara de fotoimpedancia eléctrica, mediante la integración de modelos de ingeniería de requerimientos permitieron reconocer los requisitos funcionales y no funcionales, diagramar completamente el sistema y estructurar el trabajo de forma ordenada.

Partiendo de una problemática correctamente identificada se procede a la solución de problemas específicos, mediante el diseño e implementación de subsistemas siguiendo lineamientos de arquitecturas MVC (Modelo Vista Control). El empleo de sistemas semiacoplados posibilita realizar tareas de actualización, mejora, rediseño y monitoreo del proyecto para trabajos futuros sin necesidad de implementar el sistema en su totalidad.

Se encontró que los procesos de medida son dependientes del control realizado sobre la radiación emitida. Se emplearon matrices de dispositivos LED de alta luminosidad que emiten en el infrarrojo, rojo, amarillo, verde, azul y ultravioleta. A través de la regulación de su alimentación por modulación por ancho de pulso (PWM) se obtuvo control sobre la cantidad de radiación que reciben las muestras. El sistema de control de radiación resulta entonces en un sistema 


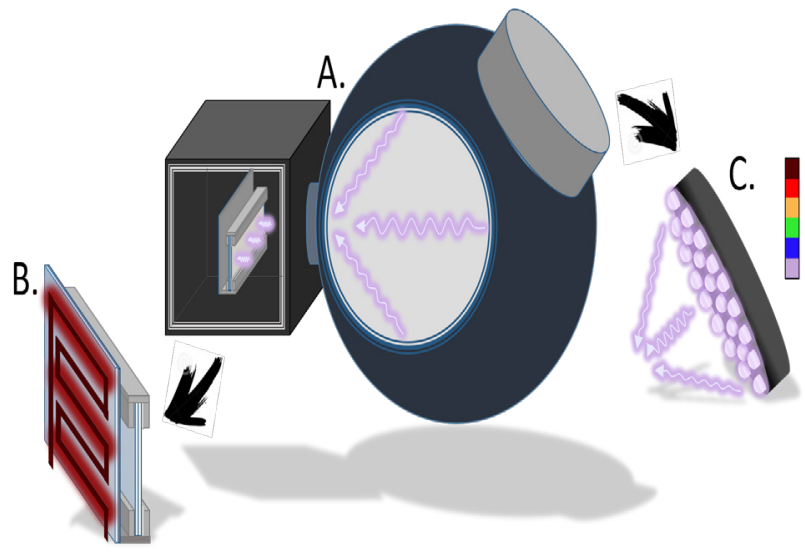

Figura 2. Diagrama de la celda de fotoimpedancia eléctrica. (A). Cámara oscura y esfera integradora. (B) Sistema eléctrico de calentamiento. (C) Matrices de LEDs con diferentes longitudes de onda.

acoplado a la cámara oscura que permite al usuario ingresar la intensidad de la radiación emitida.

Debido a las características de los encapsulados de los dispositivos LED, se hizo necesario la adición de una esfera integradora de marca LabSphere para eliminar los patrones de difracción generados en la radiación, de esta forma se logró una radiación uniforme sobre las muestras. Dentro de la celda se incorporó un arreglo de electrodos de aluminio que integra un sistema eléctrico de calentamiento para realizar las pruebas de funcionamiento de las películas.

La Figura 2 muestra el proceso de funcionamiento de la celda de fotoimpedancia eléctrica, en donde las matrices de LED de los diferentes colores radian el interior de la esfera, ésta uniformiza la luz en su interior y la transporta hacia la muestra dentro de la cámara oscura; mientras el sistema de calentamiento controla la temperatura de medida. En la Figura 3 se puede apreciar la celda de fotoimpedancia implementada y la configuración de los electrodos, se recubrieron los bordes de la película de $\mathrm{ZnO}$

con pintura de plata para mejorar los contactos. La distancia entre electrodos se ajustó para aprovechar al máximo la superficie recubierta con $\mathrm{ZnO}$ y poder evidenciar en mayor medida los efectos causados por la excitación de la muestra.

\section{Película microestructurada de $\mathrm{ZnO}$.}

En la deposición de la película se empleó una combinación de las técnicas SILAR (Succesive Ionic Layer Adsorption and Reaction) e hidrotermal, la primera encargada de la deposición de puntos de nucleación

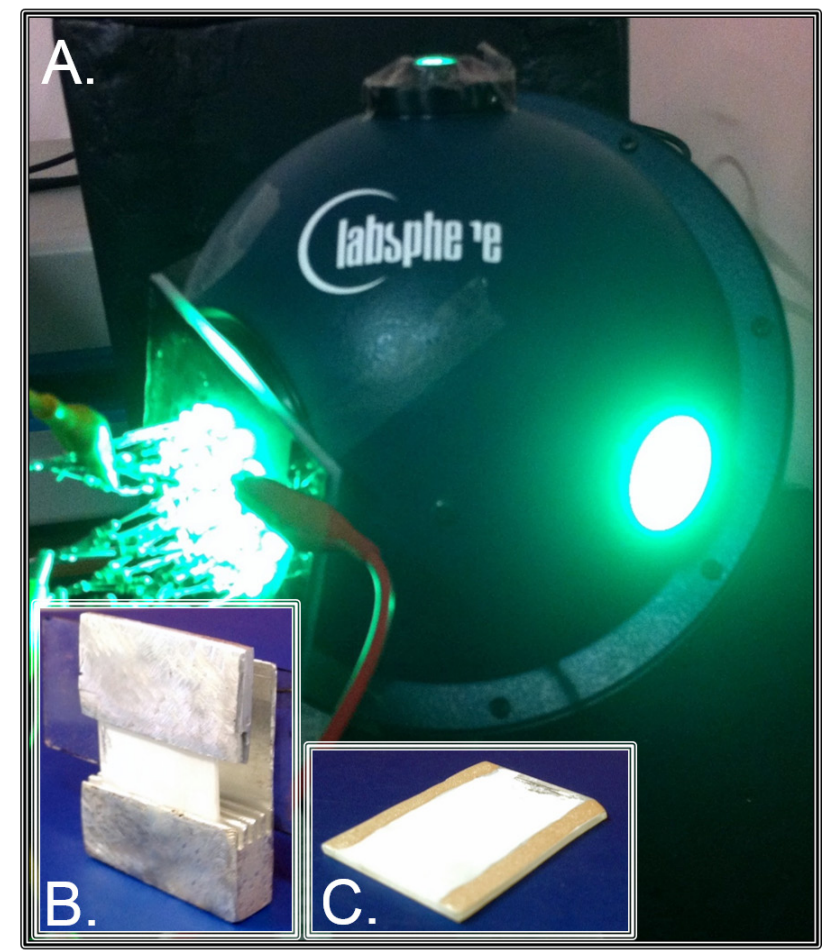

Figura 3. Celda de fotoimpedancia eléctrica.

(A) Esfera integradora con la matriz de LEDs verde. (B) Electrodos portamuestras. (C) Película de $\mathrm{ZnO}$ bordeada con pintura de plata.

de $\mathrm{ZnO}$ sobre el substrato, mientras que la segunda está directamente relacionada con el crecimiento de las varas.

Se depositaron semillas de $\mathrm{ZnO}$ sobre substratos de vidrio empleando baños químicos en una solución precursora de Sulfato de Zinc Heptahidratado ( $\mathrm{ZnSO} 4.7 \mathrm{H} 2 \mathrm{O})$ e Hidróxido de Amonio $(\mathrm{NH} 4 \mathrm{OH})$, alternado con un lavado en agua desionizada en ebullición con tiempos de inmersión de dos segundos en cada etapa y un numero de ciclos igual a 30 para el proceso. A continuación realizó el crecimiento de partículas de $\mathrm{ZnO}$, preparando una solución con agua desionizada y Acetato de Zinc Dihidratado (( $\mathrm{Zn}$ (CH3COO)2.2H2O); se agregó Hidróxido de Amonio (NH3.H2O) como agente complejante para ajustar el $\mathrm{pH}$ a un valor de 10 . Se aumentó la temperatura a 80 ${ }^{\circ} \mathrm{C}$ durante 2 horas en agitación magnética y posteriormente se dejó enfriar naturalmente. La solución y los substratos con semillas de $\mathrm{ZnO}$ fueron transferidos a un autoclave $(135 \mathrm{ml})$, sellándolo en un $80 \%$ de su capacidad volumétrica con la adición de agua desionizada y se mantuvo a una temperatura de $100{ }^{\circ} \mathrm{C}$ durante $10 \mathrm{~h}$. Después de que la solución se enfriara naturalmente se sometió la película a un tratamiento térmico de $500{ }^{\circ} \mathrm{C}$ para eliminar impurezas. En la 
Figura 4 se puede observar la micrografía SEM de la película en donde es posible apreciar la homogeneidad de las microvaras en la superficie.

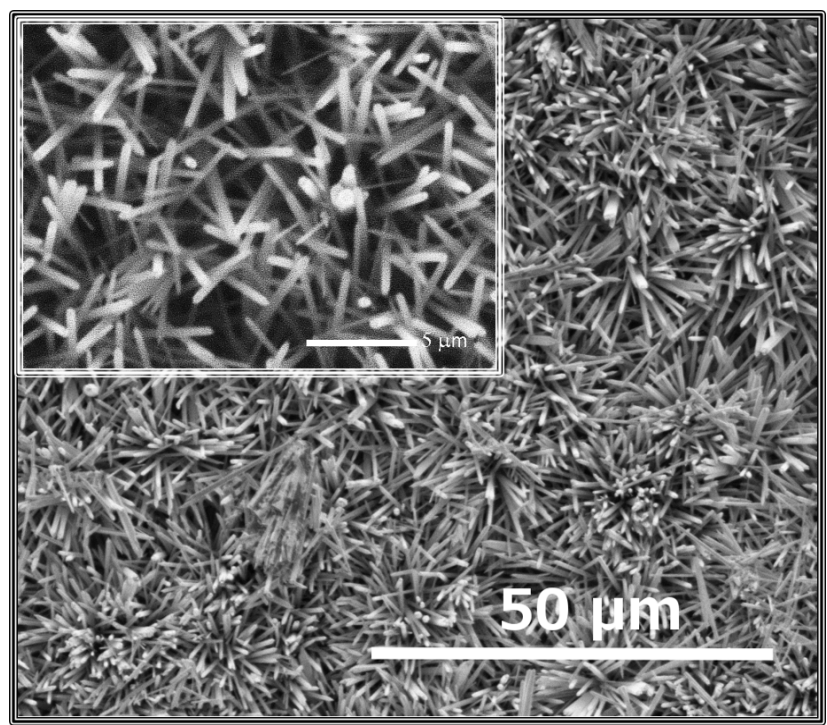

Figura 4. Micrografía SEM de la película de $\mathrm{ZnO}$. El recuadro interno muestra una escala de $5 \mu \mathrm{m}$.

\section{DETALLES EXPERIMENTALES}

En este trabajo de investigación se realizaron prácticas para determinar la eficiencia de la celda desarrollada en el estudio de propiedades optoelectrónicas de una película microestructurada de $\mathrm{ZnO}$. Se estudiaron los cambios en la resistencia eléctrica de la película por efectos de radiación UV en temperatura ambiente y a $120^{\circ} \mathrm{C}$. En ambas experiencias se llevaron a cabo medidas de espectroscopia de impedancia eléctrica a

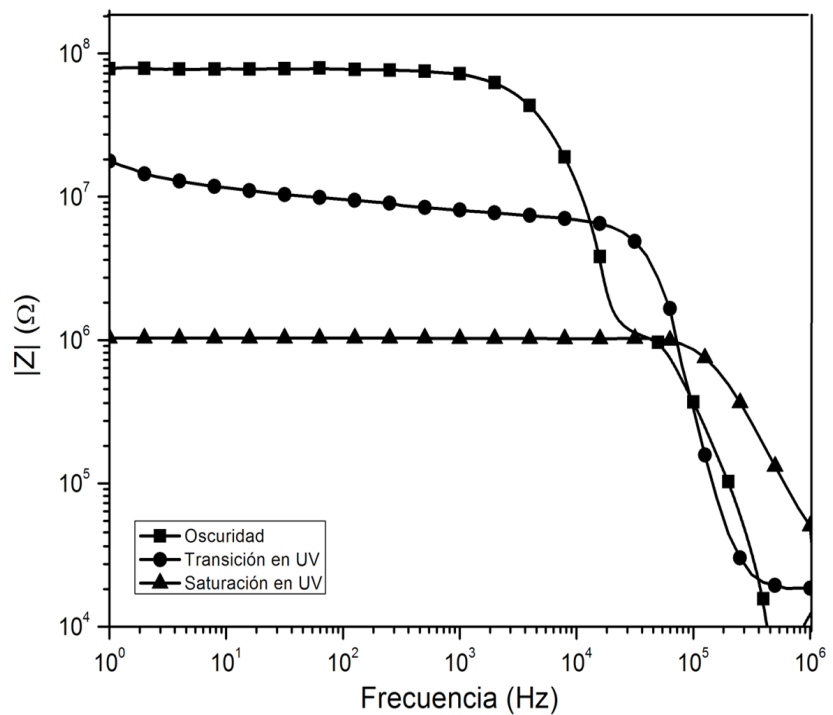

Figura 5. Diagrama de bode de los cambios de impedancia en temperatura ambiente. través del equipo SI SOLARTRON 1260 (Impedance/ Gain-Phase Analyzer) ubicando la película entre electrodos de aluminio, con una tensión de $2500 \mathrm{mV}$ en A.C. entre ellos, mientras era realizado un barrido en frecuencia desde $10 \mathrm{~Hz}$ hasta $32 \mathrm{MHz}$ para la construcción de los espectros mediante el software Zplot.

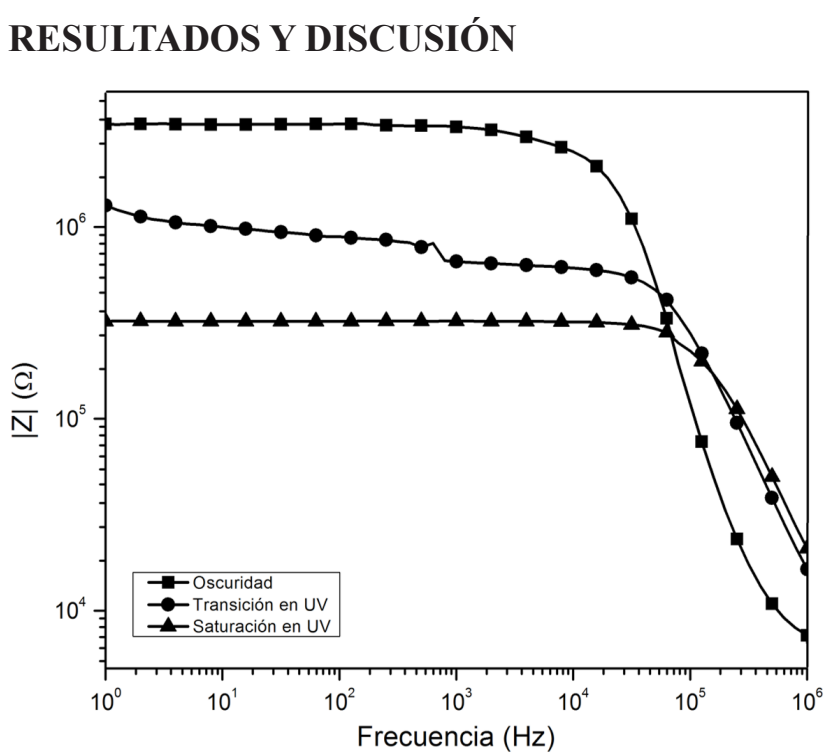

Figura 6. Diagrama de bode de los cambios de impedancia en $120{ }^{\circ} \mathrm{C}$.

Disminución de la impedancia.

En las Figuras 5 y 6 se muestran los diagramas de bode respecto a la magnitud de la impedancia de la película en temperatura ambiente y a $120^{\circ} \mathrm{C}$ respectivamente. Ambas configuraciones evidencian una disminución de la impedancia por causa de la radiación UV.

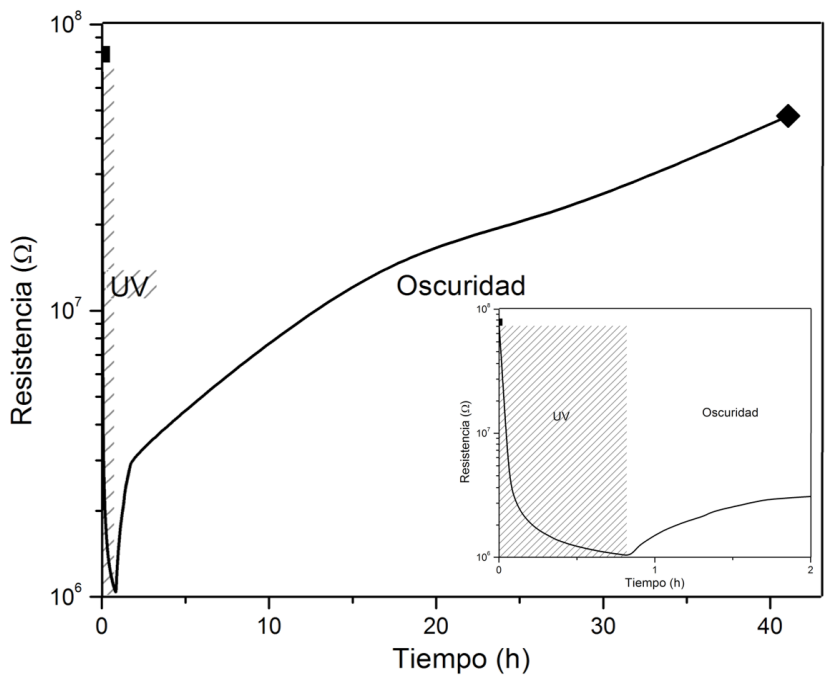

Figura 7. Gráfica del cambio de la resistencia en función del tiempo para la temperatura ambiente. 
Este efecto se asocia al aumento del número de portadores de carga por causa del rompimiento de enlaces como respuesta al bombardeo con fotones UV, cuya energía es cercana al gap de 3.17 eV calculado para la película de $\mathrm{ZnO}$.

En la película a temperatura ambiente se logró obtener una disminución de la impedancia en D.C., equivalente a la resistencia de la película, desde $7.5 \times 10^{7}$ hasta $1 \times 10^{6} \Omega$. En el estudio realizado a $120^{\circ} \mathrm{C}$ se alcanzó una disminución de la resistencia desde $2.8 \mathrm{x}$ $10^{6}$ hasta $2.6 \times 10^{5} \Omega$. La reducción de la resistencia de la película en oscuridad a $120^{\circ} \mathrm{C}$, en comparación a cuando no sufre los efectos de temperatura, se relaciona con la generación de vacancias de oxígeno por la evaporación del agua presente en la superficie.

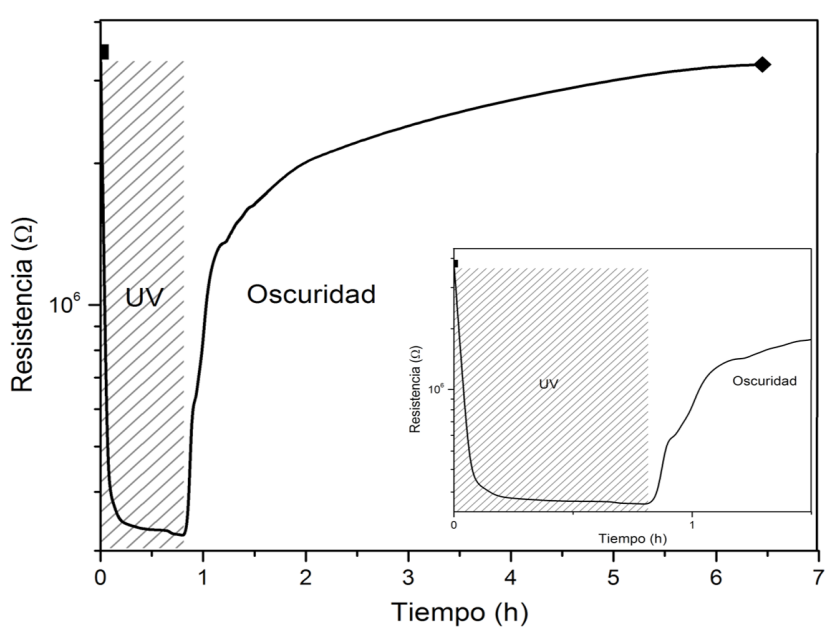

Figura 8. Gráfica del cambio de la resistencia en función del tiempo para $120^{\circ} \mathrm{C}$.

En la medida realizada a temperatura ambiente se aprecia como en oscuridad la película mantiene su impedancia constante hasta frecuencias cercanas a $10^{3} \mathrm{~Hz}$, valor que aumenta hasta la cercanía de $10^{5} \mathrm{~Hz}$ en la saturación causada por la radiación UV.

Con la temperatura de $120^{\circ} \mathrm{C}$ se haya una disminución en la diferencia de las regiones constantes de impedancia de tan solo un orden de magnitud, pasando de $10^{4} \mathrm{~Hz}$ en oscuridad a $10^{5} \mathrm{~Hz}$ en saturación; pudiéndose afirmar entonces la mejora en la estabilidad en frecuencia gracias a los efectos de temperatura.

La pendiente en las regiones de transición en ambas temperaturas está asociada al estado cambiante de la película mientras llega a su estado de saturación.
En las figuras 7 y 8 se puede apreciar el tiempo de exposición UV y de relajación en oscuridad de la película para las temperaturas ambiente y $120^{\circ} \mathrm{C}$. En ambos casos se obtuvo tiempos de saturación cercanos a los 50 minutos, no obstante la magnitud de la resistencia disminuida en ambiente es mucho mayor, por tanto se presentó una mayor velocidad de saturación en este caso.

Por otro lado, se encontró que el tiempo de relajación y regreso a su estado natural de las películas en temperatura ambiente es excesivamente alto, entre otros factores, en respuesta a la gran cantidad de resistencia disminuida. Para la película a $120^{\circ} \mathrm{C}$ se obtuvieron tiempos de relajación mucho menores debido a la disminución en la interferencia de la estabilización del material por la reducción de las moléculas de agua presentes en la superficie. En consecuencia se pudo determinar el aumento de la velocidad de relajación en la película sometida a efectos de temperatura.

\section{CONCLUSIONES}

Se logró implementar la celda de espectroscopia de fotoimpedancia eléctrica, la cual permite medir las propiedades eléctricas de películas semiconductoras en presencia de distintos tipos de radiación óptica y a temperaturas controladas. El sistema es eficiente, versátil, confiable y fortalece trabajos de investigación asociados al desarrollo de sensores ópticos.

La película microestructurada de $\mathrm{ZnO}$ presentó potencial uso como sensor UV, debido a los grandes cambios que sufre su comportamiento eléctrico en presencia de este tipo de radiación. En general el UV causó una disminución en la impedancia eléctrica de las películas, sin embargo, a pesar de que en ambiente existe un rango mayor de disminución de la impedancia, la velocidad de recuperación del estado natural es muy pequeña y no posibilita su uso como dispositivo sensor. Para solucionar este problema es posible aumentar el rompimiento de enlaces y obtener mayores reducciones de la impedancia en D.C. por efectos de temperatura, obteniéndose además un comportamiento más estable y velocidades de relajación más rápidas cuando esta se eleva por encima del punto de evaporación del agua; razón por la cual se recomienda el uso de las películas de $\mathrm{ZnO}$ en altas temperaturas para su implementación en sensores.

Tiempo de saturación y relajación. 


\section{AGRADECIMIENTOS}

Los autores agradecen al programa de Semilleros de Investigación 2013 de Colciencias, la Dirección de Investigaciones (DIMA), la Facultad de Ingeniería y Arquitectura y la Facultad de Ciencias Exactas y Naturales de la Universidad Nacional de Colombia - Sede Manizales por el apoyo en la ejecución y divulgación de este proyecto.

\section{BIBLIOGRAFÍA}

1. Sun, B. Sirringhaus, H. (2005). "Solution-Processed Zinc Oxide Field-Effect Transistors Based on Self-Assembly of Colloidal Nanorods". En Nano Letters, Vol. 5 (12), pp 2408-2413.

2. Mohanta, K. Pal, A. J. (2009) "Diode Junctions in Single ZnO Nanowires as Half-Wave Rectifiers". En Journal of Physical Chemistry C, Vol. 113 (42), pp 18047-18052.

3. Cheng, Y. Chou, S. Chang, J. (2011) "Development of flexible piezoelectric nanogenerator: Toward all wet chemical method". En Microelectronic Engineering, Vol. 88 (9), pp. 30153019.

4. Chu, S. Li, D. Chang, P. Lu, J. G. (2011). "Flexible Dye-Sensitized Solar Cell Based on Vertical ZnO Nanowire Arrays". En Nanoscale Research Letters, vol. 6 (38).

5. Zhang, L. Zhao, J. Zheng, J. Li, L. Zhu, Z. (2011) "Hydrothermal synthesis of hierarchical nanoparticle-decorated $\mathrm{ZnO}$ microdisks and the structure-enhanced acetylene sensing properties at high temperatures". En Sensors and Actuators B: Chemical, Vol. 158(1), pp. 144-150.

6. Kumar, N. Dorfman, A. Hahm, J. (2006) "Ultrasensitive DNA sequence detection using nanoscale ZnO sensor arrays” En Nanotechnology, Vol. 17(12), pp. 2875-2881.

7. Chai, G. Lupan, O. Chow, L. Heinrich, H. (2009). "Crossed zinc oxide nanorods for ultraviolet radiation detection” En Sensors and Actuators A, Vol. 150(2), pp. 184-187. 